\title{
ON SOLUTIONS FOR DERIVATIONS OF A NOETHERIAN $k$-ALGEBRA AND LOCAL SIMPLICITY
}

\author{
RENE BALTAZAR AND IVAN PAN
}

\begin{abstract}
We introduce a general notion of solution for a Noetherian differential $k$ algebra and study its relationship with simplicity, where $k$ is an algebraically closed field; then we analyze conditions under which such solutions may exist and be unique, with special emphasis in the cases of $k$-algebras of finite type and formal series rings over $k$. Using that notion we generalize a criterion for simplicity due to Brumatti-LequainLevcovitz and give a geometric characterization of that; as an application we give a new proof of a classification theorem for local simplicity due to Hart and obtain a general result for simplicity of formal series rings over $k$.
\end{abstract}

\section{INTRODUCTION}

In the classical theory of complex (or real) ordinary differential equations the Existence and Uniqueness Theorem asserts that if $D$ is an analytic vector field on $\mathbb{C}^{n}$ and $P \in \mathbb{C}^{n}$ is not a zero of $D$, then there exists an analytic map $\gamma: \Delta \rightarrow \mathbb{C}^{n}$, where $\Delta \subset \mathbb{C}$ is an open disk containing 0 , such that $\gamma(0)=P=\left(p_{1}, \ldots, p_{n}\right)$ and $\gamma^{\prime}(t)=D(\gamma(t)), t \in \Delta$.

Denote by $\mathcal{O}_{n, P}$ the ring of germs of analytic functions at $P \in \mathbb{C}^{n}$ and think of $D$ as a derivation of that ring; hence $D=\sum_{i=1}^{n} f_{i} \partial_{z_{i}}$, where $f_{i} \in \mathcal{O}_{n, P}$ and $z_{1}, \ldots, z_{n}$ are coordinates for $\mathbb{C}^{n}$. A solution as above is given by mean of $n$ functions $z_{1}(t), \ldots, z_{n}(t)$, which are analytic in a neighborhood of 0 , and such that

$$
z_{i}^{\prime}(t)=f_{i}\left(z_{1}(t), \ldots, z_{n}(t)\right), z_{i}(0)=p_{i}, i=1, \ldots, n .
$$

Since an element in $\mathcal{O}_{n, P}$ may be represented as a power series in $z_{1}-p_{1}, \ldots, z_{n}-p_{n}$, with positive convergence radius, we obtain a unique $\mathbb{C}$-homomorphism $\varphi: \mathcal{O}_{n, P} \rightarrow \mathcal{O}_{1,0}$ which maps $z_{i}$ to $z_{i}(t)$; for an element $g \in \mathcal{O}_{n, P}$ we have $\varphi(g)(0)=0$ if and only if $g(P)=0$. Notice that, conversely, such a $\mathbb{C}$-homomorphism determines a unique solution. Motivated by this remark one may consider a $k$-derivation $D$ of an abstract $k$-algebra and say what a solution of $D$ means (see Definition (1).

In [Sei1967] one finds more or less implicitly, and besides a lot of important results, the essential material to study a more general notion of solution. More explicitly, [Ha1975]

Research of R. Baltazar was partially supported by CAPES of Brazil.

Research of I. Pan was partially supported by ANII of Uruguay. 
and BLL2003] consider solutions of suitable algebraic differential equations associated to a $k$-derivation to study simplicity of local differential rings. Furthermore, such a simplicity is characterized in [Ha1974] for an interesting class of local rings. The reader may also consult [No1994, Thm 1.6.1] where there is a version of the Existence and Uniqueness Theorem for formal non autonomic differential equation systems.

In this work, which was inspired by BLL2003, we introduce and study systematically a general notion of solution associated to a Noetherian differential $k$-algebra and its relationship with simplicity, for an algebraically closed field $k$. More precisely, in $\S 2$ we analyze conditions under which such solutions may exist and be unique with special emphasis in the cases of $k$-algebras of finite type and formal series rings over $k$ (Theorem 7 ). In $\S 3$ we first generalize the simplicity criterion given in [BLL2003] and give a geometric characterization of that (Theorem 12 and Corollary 14); next we give a new proof of the classification theorem for local simplicity [Ha1974, Thm. 2] and obtain a general result for simplicity of formal series rings over $k$ (Corollary 16 and Theorem 17).

\section{Existence AND Uniqueness TheOrem}

Let $k$ be an algebraically closed field of characteristic 0 and let $R$ be a $k$-algebra. We denote by $\operatorname{Der}_{\mathrm{k}}(R)$ the $k$-vector space of $k$-derivations of $R$. If $R=k[[t]]$ we denote by $\partial_{t}$ the canonical derivation in $\operatorname{Der}_{\mathrm{k}}(k[[t]])$.

Recall that an element $D \in \operatorname{Der}_{\mathrm{k}}(R)$ extends in a unique form to a $k$-derivation in the total quotient ring of $R$ by the formula $D(a / s)=D(a) s^{-1}-a s^{-2} D(s)$; then it also extends to any localization of $R$.

Definition 1. Let $D \in \operatorname{Der}_{\mathrm{k}}(R)$ be a derivation and let $\mathfrak{p} \in \operatorname{Spec}(R)$ be a prime ideal; denote by $k(\mathfrak{p})$ the residue field of $\mathfrak{p}$. A $k$-homomorphism $\varphi: R \rightarrow k(\mathfrak{p})[[t]]$ is said to be a solution of $D$ passing through $\mathfrak{p}$ if $\varphi \circ D=\partial_{t} \circ \varphi$ and $\varphi^{-1}((t))=\mathfrak{p}$. When $\varphi(R) \not \subset k(\mathfrak{p})$ we say the solution is nontrivial.

A solution $\varphi: R \rightarrow K[[t]]$ as above, $K=k(\mathfrak{p})$, factorizes through the localization map $R \rightarrow R_{\mathfrak{p}}$ to give a solution $\varphi_{\mathfrak{p}}: R_{\mathfrak{p}} \rightarrow K[[t]]$ passing through $\mathfrak{p} R_{\mathfrak{p}}$ (of $D$ thought as a derivation $R_{\mathfrak{p}} \rightarrow R_{\mathfrak{p}}$ ).

Remark 2. A solution $\varphi$ as in the definition above is trivial if and only if it induces a monomorphism $R / \operatorname{ker} \varphi \rightarrow k(\mathfrak{p})$; this signifies $\mathfrak{p}=\operatorname{ker} \varphi$ is a maximal ideal.

If $D \in \operatorname{Der}_{\mathrm{k}}(R)$, as pointed out in [Sei1967] we may extend $D$ to an element in $\operatorname{Der}_{\mathrm{k}}(R[[t]])$ and define the exponential $R$-automorphism $e^{t D}: R[[t]] \rightarrow R[[t]]$ given by

$$
\alpha \mapsto \sum_{n=0}^{\infty} \frac{t^{n}}{n !} D^{n}(\alpha),
$$


where $D^{n}=D^{n-1} \circ D$, for $n \geq 0$, and $D^{0}=I d$. Notice that $e^{t D}$ restricts to $R$ to give a $k$-homomorphism $R \rightarrow R[[t]]$.

In the sequel we think of $D$ as an element in $\operatorname{Der}_{\mathrm{k}}(R)$ or $\operatorname{Der}_{\mathrm{k}}(R[[t]])$ according to our convenience.

Denote by $\epsilon_{\mathfrak{p}}: R \rightarrow k(\mathfrak{p})=R_{\mathfrak{p}} / \mathfrak{p} R_{\mathfrak{p}}$ the canonical map and let $\epsilon_{\mathfrak{p}} \otimes 1: R \otimes_{k} k[[t]]=$ $R[[t]] \rightarrow k(\mathfrak{p}) \otimes_{k} k[[t]]=k(\mathfrak{p})[[t]]$ be its natural extension to power series. If $D \in \operatorname{Der}_{\mathrm{k}}(R)$, then $\epsilon_{\mathfrak{p}} \circ D$ is a $\epsilon_{\mathfrak{p}}$-derivation of $R$. We have:

Lemma 3. The map $\left.\left(\epsilon_{\mathfrak{p}} \otimes 1\right) \circ e^{t D}\right|_{R}: R \rightarrow k(\mathfrak{p})[[t]]$ defines a solution of $D$ passing through $\mathfrak{p}$. Moreover, that solution is nontrivial if and only if $\epsilon_{\mathfrak{p}} \circ D \neq 0$.

Proof. For $\alpha \in R$ we have

$$
\begin{aligned}
\left(\left(\epsilon_{\mathfrak{p}} \otimes 1\right) \circ e^{t D} \circ D\right)(\alpha) & =\sum_{n=0}^{\infty} \frac{t^{n}}{n !} \epsilon_{\mathfrak{p}}\left(D^{n+1}(\alpha)\right) \\
& =\partial_{t}\left(\sum_{n=-1}^{\infty} \frac{t^{n+1}}{(n+1) !} \epsilon_{\mathfrak{p}}\left(D^{n+1}(\alpha)\right)\right) \\
& =\left(\partial_{t} \circ\left(\epsilon_{\mathfrak{p}} \otimes 1\right) \circ e^{t D}\right)(\alpha) .
\end{aligned}
$$

Clearly $\left(\epsilon_{\mathfrak{p}} \otimes 1\right) \circ e^{t D}(\alpha) \in t k(\mathfrak{p})[[t]]$ if and only if $\epsilon_{\mathfrak{p}}(\alpha)=0$, that is, if and only if $\alpha \in \mathfrak{p}$. Moreover, if for an element $\alpha \in R$ we get $\epsilon_{\mathfrak{p}}(D(\alpha)) \neq 0$, then the linear term in the power series $\sum_{n=0}^{\infty} \frac{t^{n}}{n !} \epsilon_{\mathfrak{p}}\left(D^{n}(\alpha)\right)$ does not vanish; notice that $\epsilon_{\mathfrak{p}} \circ D=0$ implies $\epsilon_{\mathfrak{p}} \circ D^{n}=0, \forall n \geq 0$. Putting all together we obtain the proof.

Remark 4. All derivations admit at least a solution passing through a given prime ideal by Lemma 3. However, as shown in Example 9, for a given derivation all such solutions may be trivial.

If $f: A \rightarrow B$ is a homomorphism of commutative rings, then $f^{*}: \operatorname{Spec}(B) \rightarrow \operatorname{Spec}(A)$ denotes the map $\mathfrak{p} \mapsto \mathfrak{p}^{c}:=f^{-1}(\mathfrak{p})$. It is continuous with respect to the related Zariski topologies. Recall that the correspondences $A \mapsto \operatorname{Spec}(A)$ and $f \mapsto f^{*}$ induce an equivalence between the categories of $k$-algebras of finite type and affine varieties over $\operatorname{Spec}(k)$, since $k$ is algebraically closed (Nullstelensatz).

Definition 5. Two k-homomorphisms $\varphi, \psi: R \rightarrow K[[t]]$ are said to be topologically equal if $\varphi^{*}=\psi^{*}$.

Example 6 . For $n \geq 1$ define the $k$-homomorphism $f_{n}: k[x] \rightarrow k[[t]]$ given by $x \mapsto t^{n}$. Then $f_{n}=f_{m}$ implies $n=m$, but $f_{n}^{*}=f_{m}^{*}$ for all $m, n \geq 1$.

We denote by $k\left[\left[x_{1}, \ldots, x_{n}\right]\right]$ the power series $k$-algebra in $n$ indeterminates. 
Theorem 7. Assume that $R$ is Noetherian and let $\mathfrak{p} \in \operatorname{Spec}(R)$. Let $D \in \operatorname{Der}_{\mathrm{k}}(R)$ be a derivation. Then:

a) D admits a solution passing through $\mathfrak{p}$ which is nontrivial if and only if $\epsilon_{\mathfrak{p}} \circ D \neq 0$.

b) Two solutions of D passing through $\mathfrak{p}$ are topologically equal; in particular, if one of these is trivial, then the other one is too.

c) If in addition $\mathfrak{m}=\mathfrak{p}$ is a maximal ideal and $R$ is either of finite type or a quotient algebra of the form $k\left[\left[x_{1}, \ldots, x_{n}\right]\right] / I$, where $I$ is an ideal of power series, then $D$ admits a unique solution passing through $\mathfrak{m}$.

Proof. The statement (a) follows from Lemma 3. (a).

To prove (b) consider a solution $\varphi: R \rightarrow k(\mathfrak{p})[[t]]$ whose existence is assured by part

First of all we note that if $\varphi(\alpha)=0$, then $\varphi(D(\alpha))=\partial_{t}(\varphi(\alpha))=0$. Hence $D(\operatorname{ker} \varphi) \subset$ $\operatorname{ker} \varphi$. Notice also that $\operatorname{ker} \varphi \subset \mathfrak{p}$.

On the other hand, since $R$ is Noetherian there exists a unique prime ideal $\mathfrak{q} \subset \mathfrak{p}$ which is maximal among the ideals $\mathfrak{a} \subset R$ satisfying $D(\mathfrak{a}) \subset \mathfrak{a}$ (see [Sei1967, §3]). From $D(\mathfrak{q}) \subset \mathfrak{q}$ we deduce $\partial_{t}(\varphi(\mathfrak{q})) \subset \varphi(\mathfrak{q})$, which is contained in $t K[[t]]$. Since $\partial_{t}$ does not stabilize nontrivial ideals then $\mathfrak{q} \subset \operatorname{ker} \varphi$. We conclude that $\mathfrak{q}$ is the kernel of any solution of $D$ passing through $\mathfrak{p}$. Since, by definition, any solution contracts $t k[[t]]$ to $\mathfrak{p}$, the statement (b) follows.

We prove (c) in the case $R=k\left[\left[x_{1}, \ldots, x_{n}\right]\right] / I$; the other case is analogous. By Lemma 3 we only need to prove the uniqueness statement.

By assumption $D$ comes from an element $D_{1} \in \operatorname{Der}_{\mathrm{k}}\left(k\left[\left[x_{1}, \ldots, x_{n}\right]\right]\right)$ such that $D_{1}(I) \subset$ I. We have $D_{1}=\sum_{i=1}^{n} f_{i} \partial / \partial x_{i}$, for some $f_{i} \in k\left[\left[x_{1}, \ldots, x_{n}\right]\right], i=1, \ldots, n$. Let $\varphi$ : $k\left[\left[x_{1}, \ldots, x_{n}\right]\right] \rightarrow k[[t]]$ be a solution of $D_{1}$ passing through a maximal ideal $M$ with $M / I=\mathfrak{m}$, and put $x_{i}(t):=\varphi\left(x_{i}\right), i=1, \ldots, n$. Hence $M=\left(x_{1}-p_{1}, \ldots, x_{n}-p_{n}\right)$ where $p_{i}=x_{i}(0), i=1, \ldots, n$.

Consider the $r$ truncation $k$-homomorphism [ $]_{r}: k[[t]] \rightarrow k[t], r=0,1,2, \ldots$, which to a power series $\sum_{i=0}^{\infty} a_{i} t^{i}$ associates $\sum_{i=0}^{r} a_{i} t^{i}$.

Recall that a power series $f \in k\left[\left[x_{1}, \ldots, x_{n}\right]\right]$ admits a Taylor development, around $p=\left(p_{1}, \ldots, p_{n}\right)$, as

$$
f(x)=\sum_{j=0}^{\infty} \lambda^{j}(x-p)
$$


where $x=\left(x_{1}, \ldots, x_{n}\right), \lambda^{0}(x-p)=f(p)$ and $\lambda^{j} \in k\left[x_{1}, \ldots, x_{n}\right]$ is a suitable homogeneous polynomial of degree $j$, for $j \geq 1$. Therefore

$$
f(x(t)) \equiv \sum_{j=0}^{r} \lambda^{j}\left(\left[x_{1}(t)-p_{1}\right]_{r}, \ldots,\left[x_{n}(t)-p_{n}\right]_{r}\right) \bmod \left(t^{r+1}\right) .
$$

Now, from $\varphi \circ D_{1}=\partial_{t} \circ \varphi$ it follows

$$
f_{i}(x(t))=\partial_{t} x_{i}(t), i=1, \ldots n .
$$

By applying (11) to $f_{1}, \ldots, f_{n}$ we deduce that the coefficient of the degree $r$ term of $\partial_{t} x_{i}(t)$ is determined by a finite number of coefficients of $f_{i}$ and the coefficients of $\left[x_{1}(t)-\right.$ $\left.p_{1}\right]_{r}, \ldots,\left[x_{n}(t)-p_{n}\right]_{r}$, for all $i=1, \ldots, n$. This proves that $\varphi$ is uniquely determined by the $f_{j}^{\prime} s$ and $p$. Since $D_{1}$ stabilizes $I$ we easily deduce that $\varphi$ factorizes through $k\left[\left[x_{1}, \ldots, x_{n}\right]\right] / I$ to give a (unique) solution of $D$ passing through $\mathfrak{m}=M / I$. This completes the proof.

Let $i$ be a nonnegative integer number. Following Ha1974] we say $R$ is $i$-singular at $\mathfrak{p} \in \operatorname{Spec}(R)$ if $\mathfrak{p} R_{\mathfrak{p}}$ can not be generated by $i+\operatorname{dim} R_{\mathfrak{p}}$ elements.

Corollary 8. Suppose that $R$ is either complete or a localization of a $k$-algebra of finite type; let $D \in \operatorname{Der}_{\mathrm{k}}(R)$. All solutions of $D$ passing through a minimal $i$-singular prime is trivial.

Proof. By [Ha1974, Thm. 1] we know $D$ stabilizes all minimal $i$-singular primes, $i \geq 0$. Theorem [7(a) implies there is a trivial solution passing through $\mathfrak{p}$. The assertion follows from $7(\mathrm{~b})$.

Example 9. Consider $R=k[x, y, z], \mathfrak{p}=(x, y)$ and the derivation $D=y \partial_{x}+x z \partial_{y}$. Hence $R / \mathfrak{p}$ may be identified with $k[z]$ in such a way that $k(\mathfrak{p})=k(z)$ is the field of rational functions in one indeterminate. by

First of all note that the solution of $D$ (passing through $\mathfrak{p}$ ) given by Lemma 3 is defined

$$
x \mapsto 0, y \mapsto 0, z \mapsto z
$$

More generally, if $f \in k(z)$, a $k$ homomorphism given by

$$
x \mapsto 0, y \mapsto 0, z \mapsto f
$$

defines a solution of $D$ passing through $\mathfrak{p}$. All these solutions are trivial (to compare with Theorem 7(b)).

On the other hand, a maximal ideal of $R$ is an ideal of the form $\mathfrak{m}_{p}=(x-a, y-$ $b, z-c)$ for some $p=(a, b, c) \in k^{3}$. The unique solution of $D$ passing through $\mathfrak{m}_{p}$ is a $k$-homomorphism $\varphi_{p}: R \rightarrow k[[t]]$ such that $\varphi_{p} \circ D=\partial_{t} \circ \varphi_{p}$. In other words, we have

$$
y(t)=\partial_{t} x(t), x(t) z(t)=\partial_{t} y(t), 0=\partial_{t} z(t),
$$


where $x(t):=\varphi_{p}(x), y(t):=\varphi_{p}(y), z(t):=\varphi_{p}(z)$.

If $\mathfrak{m}_{p} \supset \mathfrak{p}$, then $p=(0,0, c)$ and $x(t)=0, y(t)=0, z(t)=c$ satisfy (2). Otherwise, $p=$ $(a, b, c)$ with $a \neq 0$ or $b \neq 0$. Since $D^{2 m+1}(x)=y z^{m}, D^{2 m}(x)=x z^{m}, D^{2 m+1}(y)=x z^{m+1}$ and $D^{2 m}(x)=y z^{m}$, we deduce that the unique solution passing through $\mathfrak{m}_{p} \not \supset \mathfrak{p}$ is given by $z(t)=c$ and

$$
x(t)=\sum_{m=0}^{\infty}\left\{\frac{a c^{m}}{(2 m) !} t^{2 m}+\frac{b c^{m}}{(2 m+1) !} t^{2 m+1}\right\}, y(t)=\sum_{m=0}^{\infty}\left\{\frac{b c^{m}}{(2 m) !} t^{2 m}+\frac{a c^{m}}{(2 m+1) !} t^{2 m+1}\right\} .
$$

Example 10. Let $R, \mathfrak{p}$ and $\mathfrak{m}_{p}$ be as in the precedent example and let $D=\partial_{x}+\partial_{y}+\partial_{z}$. In this case the solutions passing through $\mathfrak{p}$ are of the form

$$
x \mapsto t, y \mapsto t, z \mapsto t+f
$$

where $f \in k(z)$. Analogously $\varphi_{p}(x)=a+t, \varphi_{p}(y)=b+t, \varphi_{p}(z)=c+t$ is the unique solution passing through $\mathfrak{m}_{p}$.

\section{ON SIMPLICITY FOR LOCAL NOETHERIAN $k$-ALGEBRAS}

A (Noetherian) differential ring is a pair $(R, D)$, where $R$ is a Noetherian $k$-algebra and $D \in \operatorname{Der}_{\mathrm{k}}(R)$. Given two differential rings $\left(R_{1}, D_{1}\right),\left(R_{2}, D_{2}\right)$ a $k$-homomorphism $\psi: R_{1} \rightarrow R_{2}$ is said to be a morphism of differential rings if $D_{2} \circ \psi=\psi \circ D_{1}$; we also write $\psi:\left(R_{1}, D_{1}\right) \rightarrow\left(R_{2}, D_{2}\right)$. When $\psi$ is an isomorphism we say $\left(R_{1}, D_{1}\right)$ and $\left(R_{2}, D_{2}\right)$ are isomorphic.

Notice that a solution $\varphi: R \rightarrow k(\mathfrak{p})[[t]]$ of a derivation $D \in \operatorname{Der}_{\mathrm{k}}(R)$, passing through a prime ideal $\mathfrak{p} \in \operatorname{Spec}(R)$, is nothing that a morphism $(R, D) \rightarrow\left(k(\mathfrak{p})[[t]], \partial_{t}\right)$, where one specifies the contraction of the maximal ideal. In particular, if $\psi:\left(R^{\prime}, D^{\prime}\right) \rightarrow(R, D)$ is a morphism, then $\varphi \circ \psi$ is a solution of $D^{\prime}$ passing through $\psi^{-1}(\mathfrak{p})$.

Definition 11. A differential ring $(R, D)$ is said to be simple if the unique ideals stable under $D$ are $(0)$ and $R$.

Recall that a commutative ring is said to be reduced if it has no nontrivial nilpotent elements.

Let $A$ be a commutative ring. For an ideal $\mathfrak{a} \subset A$ we put $V(\mathfrak{a}):=\{\mathfrak{q} \in \operatorname{Spec}(A) ; \mathfrak{q} \supset \mathfrak{a}\}$ : it is the Zariski closed set associated to $\mathfrak{a}$; note that for a prime ideal $\mathfrak{a}=\mathfrak{q}, V(\mathfrak{q})$ is the Zariski closure in $\operatorname{Spec}(A)$ of the single set $\{\mathfrak{q}\}$. The $\operatorname{dimension} \operatorname{dim} V(\mathfrak{a})$ of $V(\mathfrak{a})$ is $\operatorname{dim} A / \mathfrak{a}$. If $\mathfrak{b} \subset \mathfrak{a}$ one obtains $V(\mathfrak{a}) \subset V(\mathfrak{b})$ and $\operatorname{dim} V(\mathfrak{b})-\operatorname{dim} V(\mathfrak{a})$ is said to be the codimension of $V(\mathfrak{a})$ in $V(\mathfrak{b})$, denoted by $\operatorname{codim}(V(\mathfrak{a}), V(\mathfrak{b}))$.

If in addition $A$ is a $K$-algebra, where $K$ is a field, we denote by $\operatorname{trdeg}_{K} A$ the transcendence degree of $A$ over $K$. 
Theorem 12. Let $(R, D)$ be a differential ring, let $\mathfrak{p} \in \operatorname{Spec}(R)$ be a prime ideal in $R$. Assume that there exists a nontrivial solution $\varphi: R \rightarrow K[[t]]$ of $D, K=k(\mathfrak{p})$, passing through $\mathfrak{p}$. The following assertions are equivalent:

(a) $\left(R_{\mathfrak{p}}, D\right)$ is simple

(b) $\varphi_{\mathfrak{p}}$ is one to one.

(c) $R_{\mathfrak{p}}$ is reduced and the image of $\varphi_{\mathfrak{p}}^{*}$ is dense in $\operatorname{Spec}\left(R_{\mathfrak{p}}\right)$.

(d) $\operatorname{ker} \varphi$ is the unique minimal prime contained in $\mathfrak{p}$ and there is $u \in R \backslash \mathfrak{p}$ such that $u \operatorname{ker} \varphi=0$.

If, in addition, $R$ is of finite type, then $(a),(b)$ and $(c)$ are equivalent to

(e) $R_{\mathfrak{p}}$ is reduced and there is a unique irreducible component $X$ of $\operatorname{Spec}(R)$ containing $V(\mathfrak{p})$ such that $\operatorname{trdeg}_{K} \varphi(R)=\operatorname{codim}(V(\mathfrak{p}), X)$.

Proof. Since $\varphi$ is nontrivial, then $\varphi_{\mathfrak{p}}$ is too and hence $\operatorname{ker} \varphi_{\mathfrak{p}} \subsetneq \mathfrak{p} R_{\mathfrak{p}}$. As we noticed in the proof of Theorem 7, $\operatorname{ker} \varphi_{\mathfrak{p}}$ is the biggest ideal in $R_{\mathfrak{p}}$ which is stable under $D$. We deduce that $(a)$ and $(b)$ are equivalent.

On the other hand the image of $\varphi_{\mathfrak{p}}^{*}$ is dense in $\operatorname{Spec}\left(R_{\mathfrak{p}}\right)$ if and only if all element in ker $\varphi_{\mathfrak{p}}$ is nilpotent (see [AM1969, Chap. 1, Exercise. 21]), hence (b) and (c) are equivalent.

Notice that the canonical map $\lambda_{\mathfrak{p}}: R \rightarrow R_{\mathfrak{p}}$ induces a homeomorphism

$$
\operatorname{Spec}\left(R_{\mathfrak{p}}\right) \simeq\{\mathfrak{q} \in \operatorname{Spec}(R) ; \mathfrak{q} \subset \mathfrak{p}\},
$$

and consider the commutative diagram

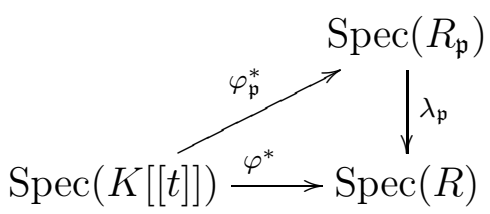

Since the images of $\varphi^{*}$ and $\varphi_{\mathfrak{p}}^{*}$ are dense in $V(\operatorname{ker} \varphi)$ and $V\left(\operatorname{ker} \varphi_{\mathfrak{p}}\right)$, respectively, we deduce that the image of $\varphi_{\mathfrak{p}}^{*}$ is dense in $\operatorname{Spec}\left(R_{\mathfrak{p}}\right)$ if and only if $V\left(\varphi_{\mathfrak{p}}^{*}\right)=\operatorname{Spec}\left(R_{\mathfrak{p}}\right)$ if and only if the right-hand side in (3) is contained and dense in $V(\operatorname{ker} \varphi)$. This is equivalent to say that $\operatorname{ker} \varphi$ is the unique minimal prime contained in $\mathfrak{p}$. Moreover, since the extension of $\operatorname{ker} \varphi$ in $R_{\mathfrak{p}}$ is $\operatorname{ker} \varphi_{\mathfrak{p}}$ we easily deduce that $(c)$ and $(d)$ are equivalent. This completes the first part of the proof.

Now suppose that $R$ is of finite type. Hence $\operatorname{dim} R / \operatorname{ker} \varphi=\operatorname{trdeg}_{k} \varphi(R)=\operatorname{trdeg}_{K} \varphi(R)+$ $\operatorname{dim} R / \mathfrak{p}$. In other words

$$
\operatorname{trdeg}_{K} \varphi(R)=\operatorname{codim}(V(\mathfrak{p}), V(\operatorname{ker} \varphi)) .
$$


If we assume that assertions $(a)$ to $(d)$ hold, then $X=V(\operatorname{ker} \varphi)$ is the unique component of $\operatorname{Spec}(R)$ containing $V(\mathfrak{p})$. From (4) we know that component has the correct dimension, then $(e)$ holds.

Conversely, assume that $(e)$ holds and notice that $V(\operatorname{ker} \varphi)$ is an irreducible closed set in $\operatorname{Spec}(R)$, which contains $V(\mathfrak{p})$. Then (4) implies $X=V(\operatorname{ker} \varphi)$, from which it follows that $\operatorname{ker} \varphi$ is the unique minimal prime contained in $\mathfrak{p}$. From (3) we deduce, as before, that the image of $\varphi_{\mathfrak{p}}^{*}$ is dense in $\operatorname{Spec}\left(R_{\mathfrak{p}}\right)$, i.e. all element in $\operatorname{ker} \varphi_{\mathfrak{p}}$ is nilpotent. Thus $\varphi_{\mathfrak{p}}$ is one to one which completes the proof.

Example 13. Locally nilpotent derivations. Let $(R, D)$ be a differential ring where $D$ is locally nilpotent, i.e. for each $a \in R$ there exists a positive integer $n$ such that $D^{n}(a)=0$. Suppose that $R$ is a (Noetherian) local ring with maximal ideal $\mathfrak{m}$. Let $\ell$ be the minimum positive integer such that $D^{\ell}(\mathfrak{m}) \subset \mathfrak{m}$. Hence $D$ stabilizes the nonzero ideal generated by $D^{\ell-1}(\mathfrak{m})$.

Suppose $\ell>1$ and consider a nontrivial solution of $D$ passing through $\mathfrak{m}$. From Theorem 7 and its proof we know that the kernel of such a solution coincides with the biggest ideal $\mathfrak{q} \subset \mathfrak{m}$ which is stable under $D$. Using the solution given by Lemma 3 we deduce

$$
\mathfrak{q}=\left\{a \in R ; D^{i}(a) \in \mathfrak{m}, i=0, \ldots, \ell-1\right\} .
$$

By using theorem 12 we conclude that $(R, D)$ is simple if and only if one of the following equivalent conditions holds:

- there exist $a_{1}, \ldots, a_{s} \in R$ and $x_{1}, \ldots, x_{s} \in \mathfrak{m}$ such that $\sum_{i=1}^{s} a_{i} D^{\ell-1}\left(x_{i}\right)=1$.

- $a \in \mathfrak{m}, D(a) \in \mathfrak{m}, \ldots, D^{\ell-1}(a) \in \mathfrak{m}$ imply $a=0$.

The following corollary generalizes the results in [BLL2003, §1] (see Remark 15).

Corollary 14. Let $R$ be a $k$-algebra of finite type without zero divisors and let $\mathfrak{p} \in$ $\operatorname{Spec}(R)$. If $D \in \operatorname{Der}_{\mathrm{k}}(R)$ is a derivation such that $D(\mathfrak{p}) \not \subset \mathfrak{p}$, then there is at least a nontrivial solution $\varphi: R \rightarrow K[[t]]$ passing through $\mathfrak{p}$ and the following assertions are equivalent:

(a) $\left(R_{\mathfrak{p}}, D\right)$ is simple.

(b) $\varphi_{\mathfrak{p}}$ is one to one.

(c) the image of $\varphi_{\mathfrak{p}}^{*}$ is dense in $\operatorname{Spec}\left(R_{\mathfrak{p}}\right)$.

(d) $\operatorname{trdeg}_{K} \varphi(R)=\operatorname{codim}(V(\mathfrak{p}), \operatorname{Spec}(R))$.

Proof. Since $D(\mathfrak{p}) \not \subset \mathfrak{p}$ implies $\epsilon_{\mathfrak{p}} \circ D \neq 0$ the existence of a nontrivial solution passing through $\mathfrak{p}$ is assured by Theorem 7 . Taking into account that in the present case $R$, and then $R_{\mathfrak{p}}$, have no zero divisors, the corollary follows readily from Theorem 12 . 
Remark 15. If $R=k\left[x, y_{1}, \ldots, y_{r}\right], \mathfrak{p}$ is the maximal ideal $\mathfrak{m}=\left(x-\alpha, y_{1}-\beta_{1}, \ldots, y_{r}-\beta_{r}\right)$, $\left(\alpha, \beta_{1}, \ldots, \beta_{r}\right) \in k^{r+1}$, and $D \in \operatorname{Der}_{\mathrm{k}}(R)$ is a derivation, then $D(\mathfrak{m}) \not \subset \mathfrak{m}$ if and only if $D=g \partial_{x}+\sum_{i=1}^{r} f_{i} \partial_{y_{i}}$, where $g, f_{1}, \ldots, f_{r} \in k\left[x, y_{1}, \ldots, y_{r}\right]$ are polynomials not all of them belonging to $\mathfrak{m}$. From the unique nontrivial solution for $(R, D)$, passing through $\mathfrak{m}$, we obtain a solution $\varphi_{\mathfrak{m}}$ of $\left(R_{\mathfrak{m}}, D\right)$. If we put $x(t):=\varphi_{\mathfrak{m}}(x), y_{i}(t):=\varphi_{\mathfrak{m}}\left(y_{i}\right), i=1, \ldots, r$, then we may read Corollary 14 as saying $\left(R_{\mathfrak{m}}, D\right)$ is simple if and only if $x(t), y_{1}(t), \ldots, y_{r}(t)$ are transcendent over $k$.

The second part of the next result is essentially [Ha1974, Thm. 2].

Corollary 16. Let $S$ be a Noetherian local $k$-algebra with maximal ideal $\mathfrak{m}$ and let $D \in$ $\operatorname{Der}_{\mathrm{k}}(S)$. Then $(S, D)$ is simple if and only if there is a one to one solution passing through $\mathfrak{m}$. In particular, $(S, D)$ is isomorphic to a differential ring $\left(S_{0}, D_{0}\right)$, where $S_{0}$ is a $k$-subalgebra of $K[[t]], K=k(\mathfrak{m})$, which is stable under $\partial_{t}$ and $D_{0}:=\left.\partial_{t}\right|_{S_{0}}$.

Proof. Note that simplicity implies $D(\mathfrak{m}) \not \subset \mathfrak{m}$, from which we know $D$ admits nontrivial solutions (Theorem 7(a)). By Theorem 12 we deduce $S$ is $D$-simple if and only if there exists a one to one solution, say $\varphi: S \rightarrow K[[t]]$. For the rest of the proof we take $S_{0}=\varphi(S)$, and the rest of the assertion is essentially trivial.

For the completion of a $k$-algebra of finite type, with respect to a maximal ideal, simplicity is quite rare. In fact, suppose $R=k\left[\left[x_{1}, \ldots, x_{n}\right]\right]$ and let $\varphi: R \rightarrow k[[t]]$ be the unique solution passing through $\mathfrak{m}=\left(x_{1}, \ldots, x_{n}\right)$, associated to a derivation $D$. Corollary 16 implies $\operatorname{ker} \varphi \neq 0$ unless $n=1$ and $D=f \partial_{x_{1}}$ for some $f \in k\left[\left[x_{1}\right]\right]$ with $f(0) \neq 0$. More generally, we have:

Theorem 17. Let $R$ be the quotient of $k\left[\left[x_{1}, \ldots, x_{n}\right]\right]$ by an ideal I. If $D \in \operatorname{Der}_{\mathrm{k}}(R)$, then $(R, D)$ is simple if and only if $D$ lifts to a derivation $\widehat{D} \in \operatorname{Der}_{\mathrm{k}}\left(k\left[\left[x_{1}, \ldots, x_{n}\right]\right]\right)$ which admits a unique solution $\widehat{\varphi}: k\left[\left[x_{1}, \ldots, x_{n}\right]\right] \rightarrow k[[t]]$, passing through the maximal ideal $\left(x_{1}, \ldots, x_{n}\right)$, such that $\operatorname{ker} \widehat{\varphi}=I$.

Proof. Note that each element in $\operatorname{Der}_{\mathrm{k}}(R)$ comes from an element in $\operatorname{Der}_{\mathrm{k}}\left(k\left[\left[x_{1}, \ldots, x_{n}\right]\right]\right)$ which stabilizes $I$ and that under this correspondence we obtain compatible solutions. Recalling that two solutions passing through the maximal ideal have the same kernel (Theorem 7b) the result follows from Corollary 16.

\section{REFERENCES}

[AM1969] M. F. Atiyah, I. G. Macdonald, Introduction to Commutative Algebra, Addison-Wesley Publishing Company, Massachusetts, 1969.

[BLL2003] P. Brumatti, Y. Lequain and D. Levcovitz, Differential simplicity in Polynomial Rings and Algebraic Independence of Power Series, J. London Math. Soc. (2) 68 (2003), pp. 615-630.

[Ha1974] R. Hart, Derivations on commutative rings, J. London Math. Soc. (2), 8 (1974), pp. 171-175. 
[Ha1975] R. Hart, Derivations on Regular local rings of finitely generated type, J. London Math. Soc. (2), 10 (1975), pp. 292-294.

[No1994] A. Nowicki, Polynomial derivations and their rings of constants, at http://www-users.mat.umk.pl/ anow/ps-dvi/pol-der.pdf

[Mat] H. Matsumura, Commutative Ring Theory, Cambridge University Press, 1989.

[Sei1967] A. Seidenberg, Differential ideals in rings of finitely generated type, American Journal of Mathematics, Vol. 89, No. 1 (1967), pp. 22-42. 